\title{
Health Status at the Time of Birth (Health by Birth Analyzing Through Finger Impressions)
}

\author{
Venkateswara Rao Madduru- Fingerprint Analyzer \\ Finger Print Bureau, CID. Andhra Pradesh. India
}

\begin{abstract}
I am an expert in Dermatoglyphics i.e. study of fingerprints. In this discipline, I have an experience of 28 years. My research work is on the formation of patterns on the first phalange of all the hand fingers and also on the skin of palms. By authenticating the finger impressions and its nature of forming ridge characteristics (ridges as well as its patterns), I have understood that each finger impression is working as an index to an organ which in turn leads to the analysis of organ development and its condition. For declaring this statement, I have verified thousands of finger impressions formed at first phalange of fingers.
\end{abstract}

Key words: Fingerprint, Health by Birth, Friction Skin, Analyzing, Human Organs, Child Health Status.

\section{Evolution Of Finger Impressions}

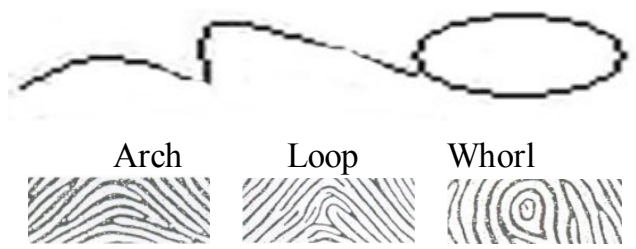

Fingerprint pattern, which is formed at first phalange of finger is in close association with the evolution of Organs i.e., Organ development at womb is simultaneously reflected in the first phalange. There are parallel changes in the pattern as well as organ development. While analyzing the finger impressions the development of Organs and its condition can be decided. Fingerprint pattern formed on the bulb of the first phalange of the finger acts as a reference of the Organs.

Normally Fingerprints can be classified broadly into three family types and they are: 1.Arch, 2.Loop and 3. Whorl. All Finger Impressions come under these families only.

The finger impressions are

Arch

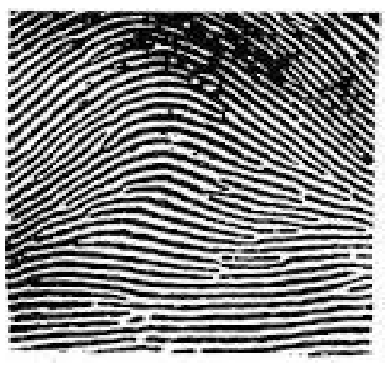

Loop

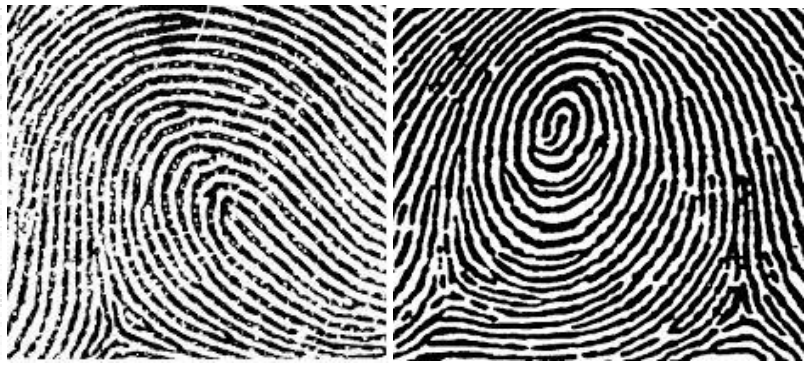

Every pattern must be one of Arch family or Loop family or Whorl family.

Latest developments in the field of science have stated that each finger is associated with various human Organs. At the time of birth we can be aware of the human organ development and its issues by means of fingerprint patterns formed in the first phalange of fingers. Through this linking, I have accomplished a number of tasks at our place and given some suggestions for maintaining the Organs. 
There are seven health groups of people in the world which are mentioned below:

Group -1: 15 to 20 Percent of People have no resistance against virus.

(Having the patterns of Arches)

Group-2: 65 to 70 Percent of People have some resistance against virus.

(Having the patterns of Loops)

Group-3: 15 to 20 Percent of People have resistance against virus.

(Having the patterns of Whorls)

Group-4: between Group - 1 and 2

(Having the patterns of combined Arches and Loops)

Group-5: between Group-2 and 3

(Having the patterns of combined Loops and Whorls)

Group-6: People with under developed Organs (Body Organs Construction)

(Having the patterns of Arches formed only straight flattered Lines)

Group-7: People with incorrect organ development (have the patterns of any with converging ridges).

Organ Strength depends upon the size also. If a person have heavy body, the organ size and strength is lower than the body requirement. So the person faces health problem and he/she will suffer form ill health frequently. So the Organs strength and body weight must be support each other.

Can we know the size and strength of the Organ?

Yes, there is a way by verifying/analyzing the finger impressions we can understood the strength of the organ whether it is fit for the body or not.

Now-a-days people are not maintaining their Organs properly. Organs as per the body and vice-versa must be maintained. Therefore, the analysis of finger impressions will be extremely useful to the Human to maintain Organs properly.

If a person knows which strength group he/she belongs to, then he/she will take care of their health. This is achievable by verifying finger impressions. Body surveillance depends mainly on Brain, Bladder, Kidneys, Pancreas, Heart, Stomach, Liver, Spleen and Lungs. The strength and size of these Organs can be analyzed through finger impressions.

Through analyzing the finger impressions from right thumb to left little we can understand the evolution (construction stages) of major Organs.

\section{Fingerprint Science}

The Pioneer's in Fingerprint Science had given valuable and evergreen thesis to the World. Through the fingerprints science and with the latest technology, number of software programs has been developed to fulfill human requirements. One of such requirements is to identify a person through finger impressions in an easier and cost efficient method.

This finger impression technology can be used in many areas one such application is in human transportation.

Finger impressions are formed in the first phalange of a finger from right thumb to right little and from left thumb to left little, 10 fingers having ridge characteristics (UNIQUE) with patterns. With these unique ridge characteristics we can analyze the health by birth.

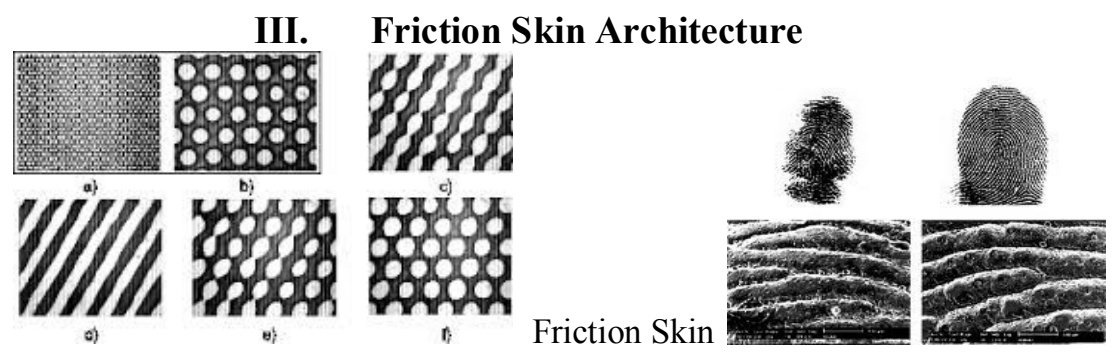

Friction Skin Ridges at palm and foot surface is created, especially the friction skin at (finger impressions) first phalange of fingers is linking with the main human Organs, they are reference of the Brain, Bladder/Sex Organs, Kidneys, Pancreas, Heart, Stomach, Liver, Spleen and Lungs. Each and every part of our body is essential and necessary for daily activities, there is no waste part created in our body. Some Organs are 
dependent on other Organs and some are independent. So it is very important to know each and every internal Organ's functions and discharges.

Now we are discussing mainly discharges of human Organs and its functions.

The internal Organs as part of their daily functions the discharge is one of the function and releases wastage from Organ.

Now let us discuss about some discharges that are useful to our body, Ex: Hair, Nail, etc., these discharges are from are playing an important role.

What about the discharges from Organs?

The Organ has the function of discharges. The discharges from Internal Organs moving towards end of the body and making some special Skin known as Friction Skin. With these discharges the Skin (Friction skin which is available on hands and foots) is formed with Ridges and Furrows doing an important role as just they are references of the Organs.

On analyzing these ridges we can understood

1. How the Organs was constructed.

2. How is Organs condition?

3. How Organ is linked with First Phalange of Fingers.

The Friction skin which is formed on palmer surface of hand and foot is made up of discharges from Important Human Organs.

The discharges like fat, water with some special conditional material is travelling through neural system and ending at palm and foot surface. Endorsing this special material the friction skin is formatted with ridges and furrows. This is a continuous process, so this skin is different from other skin of the Body. In our daily work we are removing some of this discharged material from friction skin by the way of cleaning hands and handling work.

On research the following is noted:

1. Discharges (like fat and other fatty acids with water) from internal organs travelling directly or through neural system reaches at palm surface of hand and foot, discharged out through sweat pores (the collection of sweat pores as ridges).

2. Any disturbances found in pattern of ridges, indicate improper discharges. Causation of disappearing ridges thus indicates a malfunctioning organ.

3. From this we can understand the organ's condition either is either weak or strong.

How much capacity does it have to carry out daily work?

The friction skin, which is related to the organs, is in more heat than the other Skin heat.

The friction skin, which is related to the organ, might have lost the original status of ridges.

Why did the ridges lose their original status?

If any organ lost has lost strength or any disturbance in daily work, it can't perform properly. So the discharges from the respective organ may be lesser or nil. Hence the pores in ridges don't get daily discharges, the ridges disappear slowly. Through this we can understood that the organ is in problem.

The human organs strength and its working depends upon

a. Heat (every organ releases heat during daily work).

b. Discharges (every organ discharges fat and other material. The friction skin endorses these discharges).

c. Friction skin with its sweat pores composes ridges of proper lines

d. Patterns (group of ridges).

e. Patterns (Proper composing ridges/improper composing ridges).

f. Heat (every Organ works as an engine and releases heat on his daily work).

g. Discharges (every Organ releases fat and other material. These discharges come out side of the body through PORES available at friction skin).

h. Friction Skin composing of Ridges and Furrows. 


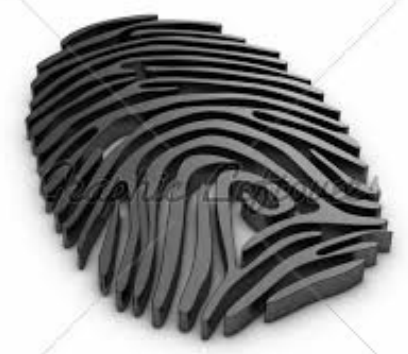

Elevations are ridges

Depressions are furrows

i. $\quad$ Ridges composing of Sweat Pores.

j. Patterns (Proper composing of ridges/In proper composing of ridges).

Proper composing of ridge patterns

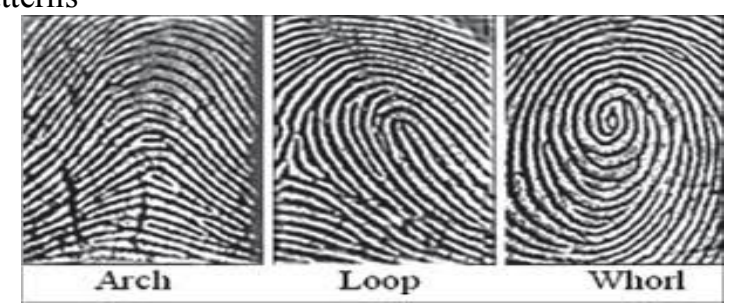

In proper composing of ridge patterns
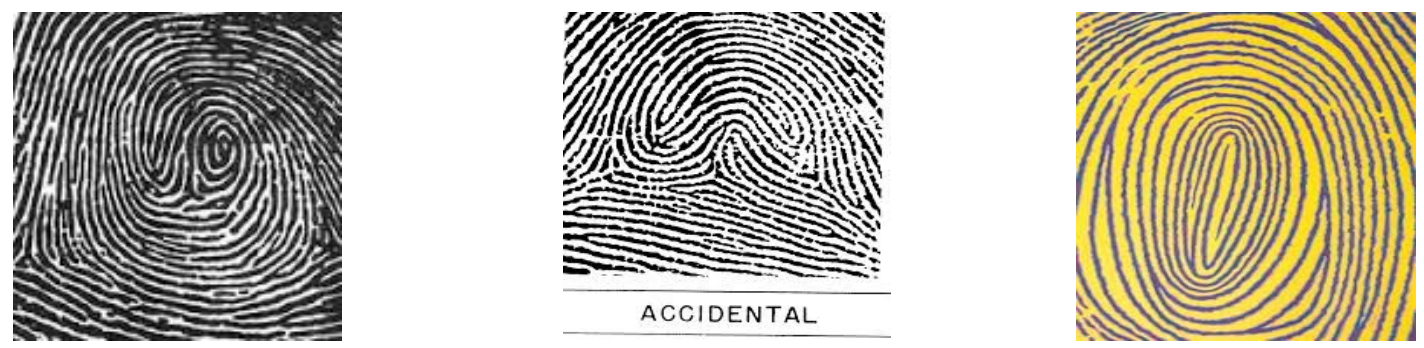

4. The Root from internal organs to sweat pores and vice versa.

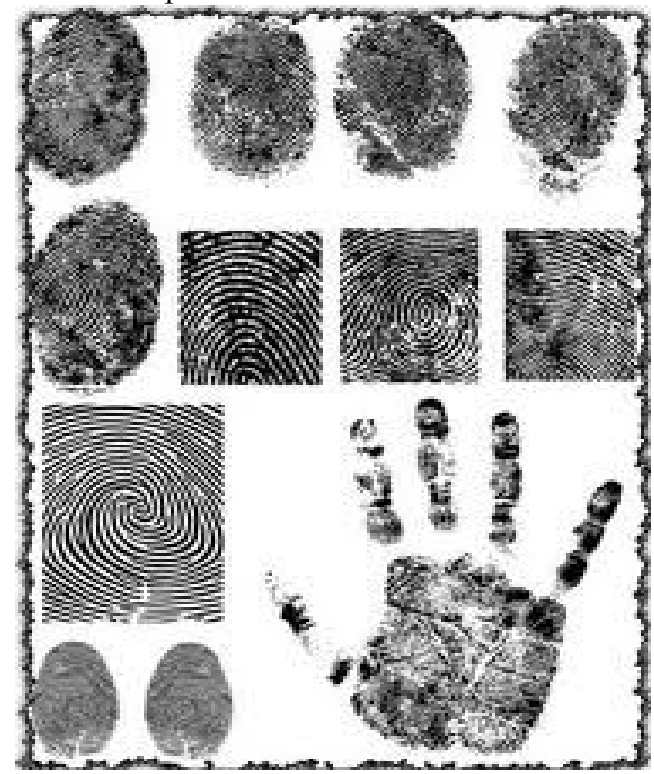

So far we discussed about Friction Skin how and why it is created. 
Next we are going to discuss about the relationship between friction skin and human organs. The friction skin EXCLUSIVELY on hand's palm surface of the first phalange of fingers is showing patterns and working as an index of organs i.e. index of the human internal organs like Brain, Bladder/Sex Organs, Kidneys, Pancreas, Heart, Stomach, Liver, Spleen and Lungs. By verifying the finger impressions and its patterns, ridge converging, ridges flowing and grouping of characteristics, we can understand the strength of the organs and any problems.

There is a relationship between the organ and this portion of the friction skin. With this friction skin portion we can understand how the development of organ. Directly we can't assess organ but we can assess organ easily with the help of this friction skin. We can examine friction skin and gain knowledge of respective internal organ.

If we go through the anatomy of heart, its pericardium the center part of the heart is covered with some layers. Likewise other organ's central portion is also covered with layers.

Arch Loop

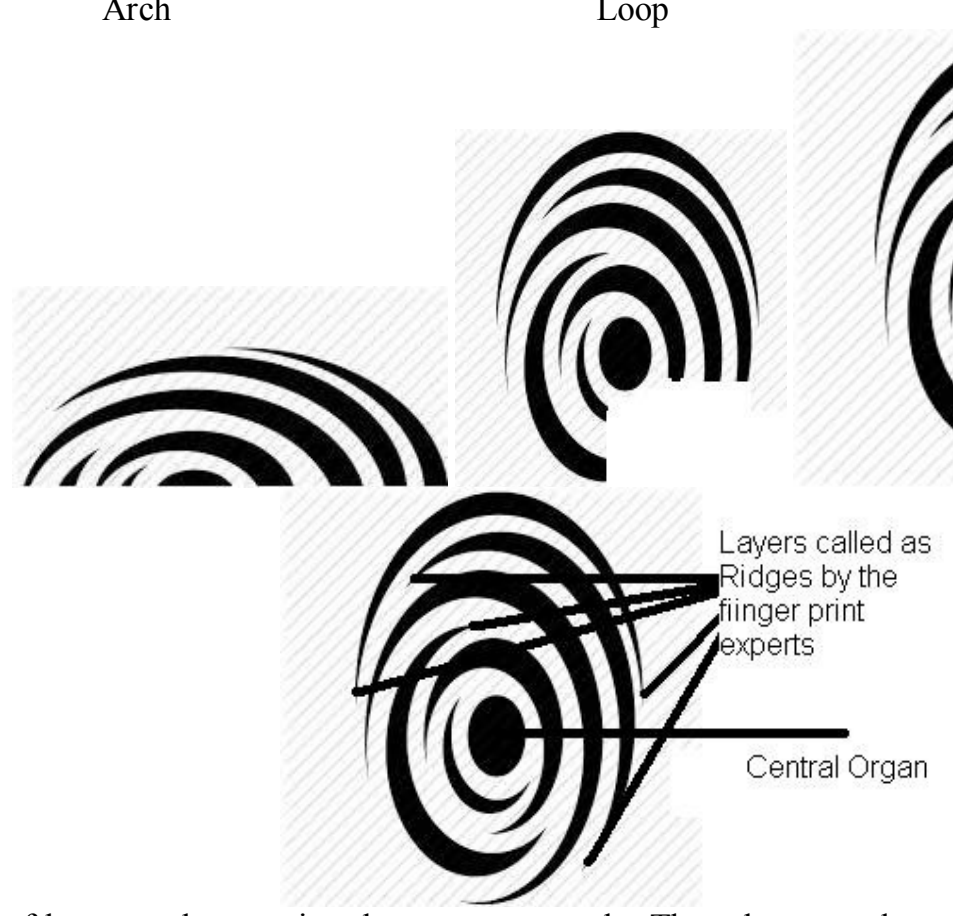

Whorl

with some of layers and protecting the organs properly. These layers and construction information we can get through related finger impression. Protection (layers around the Central Organ) the strength (with how many layers the Central Organ was covered) and the thickness of the layers also we can analyze.

Energy is needed by every organ to perform its functions properly. If there is low supply of energy than the requirement for a long time then the organ faces problem and felt as illness. There is need to take external energy. When pancreas is unable to produce required insulin (which is needed to convert sugar in food to make energy), it is called Diabetic. To compensate we inject insulin.

To avoid some extent we maintain food habits that is Limited Food Maximum Times. Pancreas releases insulin immediately after taking food. The balance of Insulin Production and requirement of Insulin to Organs should be maintained through food habits. We have to know the how much Insulin required by each Organ. This will approach by verifying finger impressions.

Arch pattern - linked indexed organs taking less energy. So the organ has less strength.

Loop pattern - linked indexed organs taking more energy than Arch pattern - linked indexed organs.

So the organ has general strength.

Whorl pattern- linked indexed organs taking more energy than Loop pattern - linked indexed organs.

So the organ has maximum strength. 
These patterns are found at first phalange of fingers (linked with organs). Through finger impressions we can analyze, what is the much energy level required.

There is no problem, if production of energy and requirement of energy (by organ) is equal.

However there is a problem, if production of energy is lower than requirement of energy (by organ), then to maintain energy level we have to take external energy similar to the case of injecting insulin (Diabetic).

\section{So all this can be understood (without blood test) by analyzing the finger impressions.}

How are the organs and how much energy they require can be analyzed through finger impressions.

Inside the womb along with organ evaluation, the reference finger friction skin develops stage-by-stage hand-in-hand. The reference finger friction skin stops and secures as organ evolution stops.

The organ central part is fixed with the functions. These functions should be worked without obstructions. All these functions are depending upon the construction of organ and its layers.

1. How many layers do cover the central part of the organ?

2. The strength of layers and extruding capacity.

3. How the layers are protecting from the disease and other attacks.

4. The Thinness of the layers.

The central organ and its functioning capacity depend upon the central organ defense system.

Central organ defense system i,e the layers covering central organ is referred here as defense system.

The layers covering by the central Organ is known as defense system.

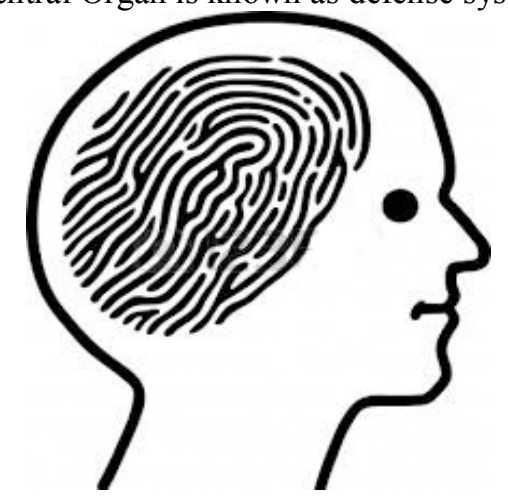

The layers

1. How the central organ is covered. (Defense System)

2. With how many layers central organ is covered.

3. The layers and their strength.

Ex: The central organ covered by layers (defense system) is as follows:

Defense system is the central organ's protection from harmful infections.

First: Arch Defense System

In this system there isn'tmuch protection for central organ. These organs are more prone to deceases and also the organ's condition is weak. There are number of ways to disturb the functioning of central organ.

Some of the organs have less protection from harmful infections.

Stage -1

In organ development maturing stages, this is the first stage.

Arch Defense System

Arch has less strength.

As said these organs have limited protection from harmful bacteria. 
Stage-2

Loop Defense System

With this system the central organ portion is safe in three ways.

But there is only one way to disturb the central organ portion.

This limited protection from harmful infection depends upon the covering layers. If there are fewer layers, represents low strength and vice-versa. In the reference finger impression we can find ridges from delta to core. The ridge count and layers represent organ layers covering the central organ.

This general protection from harmful Bacteria is depends upon the covering of layers. If low layers low strength high layers more strength than low layers. In the reference finger impression we can find ridges from delta to core. The ridge count and Organ layers (covered by the central Organ) are one and the same.

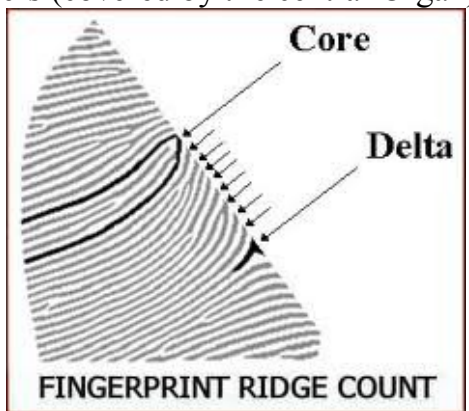

Stage-3

Through ridge count we can decide the strength of the Organ.

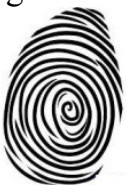

Whorl Defense System:

Organs have full Protection from harmful Bacteria.

With this system the Central Organ is in safety position. Central Organ is totally covered with the layers just like a Ball. Hence there is no way to disturb Central Organ.

This full protection from harmful Bacteria is depends upon the covering of layers. If low layers low strength high layers high strength. In the reference finger impression we can find ridges from delta to core.

Through ridge count we can decide the layers count of the Organ.

The full protection same to same as mentioned in Loop type of patterns. Here the Circle around the Central Organ is decides the protection.

There is a facility to know the internal organ structure and its defense system through finger impressions and its ridge formation vice versa the finger impressions and its ridge formation is linked with the organ structure and its defense system. So directly we can't see the organs, but we can see the finger impressions and its ridge formation directly by obtaining the finger impressions. The information about the organ is scripted in friction skin. By analyzing the finger impressions and friction skin we can understand the organs condition.

The evolution of organ vice versa evolution of Finger Impressions each one is based on one another. At womb (evolution) organ and finger impression simultaneously develops day to day and stops when the baby is born.

There are various organ evolution levels. At any moment when the baby is born the evolution will stop.

The baby development at womb and the baby development outside of the womb are different. 
The evolution may be stop after completing the organ structure or may be stops before some stages of completing the organ evolution.

We can't say if the development of organs follows formation of patterns (at First phalange of fingers) or vice-versa. Both development and formation depends on one to another. The pattern, which is on external surface of the body, indicates the organs structure and reveals its nature.

The external finger impression is helps in analyzing hidden organ.

The evolution of Organ vice versa evolution of Finger Impressions each one is base with one another. At Womb (evolution) Organ and finger impression simultaneously develops day to day and stops when the baby was out.

These development stages are various. At any moment when the baby comes out the development stage will stop. The baby development at Womb and the baby development at outside of the body are different.

The development stages may be stops after completing the Organ Structure are may be stops before stages of completing the Organ Structure.

We can't say if the development of organs follows formation of patterns (at First phalange of fingers) or viceversa. Both development and formation depends on one to another. The pattern, which is on external surface of the body, indicates the organs structure and reveals its nature.

The external finger impression is helps in analyzing hidden organ.

\begin{tabular}{|l|l|l|}
\hline S\# & Pattern & Organ Condition \\
\hline 1. & Arch & Arch Defense System \\
\hline 2. & Loop & Loop Defense System \\
\hline 3. & Whorl & Whorl Defense System \\
\hline
\end{tabular}

Other stages which signify problem:

- Any pattern with converging ridges

- Any pattern in anti-direction to the flow of ridges

- Any pattern which uneven group of ridge characteristics

So the finger impression is working as an index of relative organ and establishes its nature.

Natural: Naturally the development of organ and development of its reference at friction skin i.e. finger impression are one and the same. Organ is compound of flush, nerves and other.

The finger impressions are compound of pattern, ridges and ridge characteristics.

The built of central organ can also be analyzed through finger impression of concerned organ (reference at first phalange of finger). Everybody have the same organs. But, no two can have identically built organs. Nature can do two things but can't produce duplicate. The organ may be difference in construction, may be difference in volume or may be difference in view. Anything can differ from one to one. All this can be observed through finger impressions. Let us discuss about natural development of organ.

There are three stages

1. Arch defense system

2. Loop defense system

3. Whorl defense system.

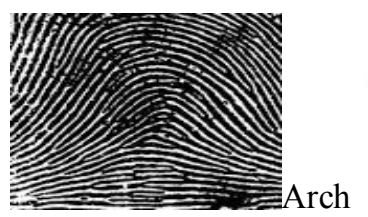

Right Side of the Organ

Arch

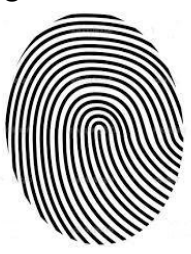

Loop 


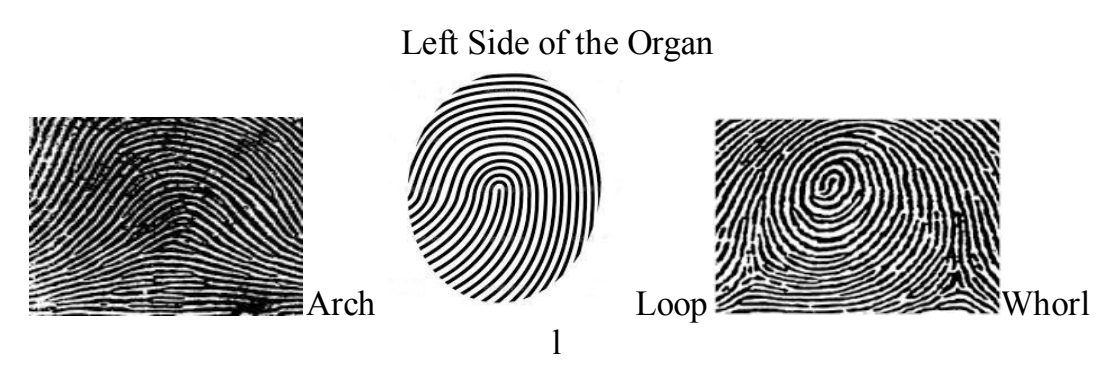

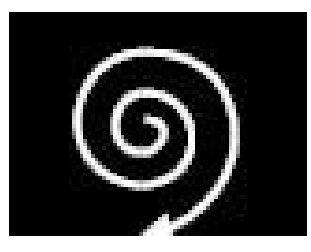

Clock wise formation on left side finger impressions

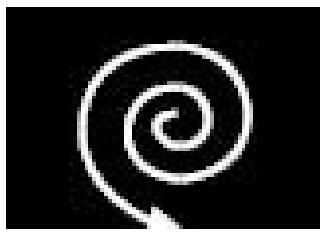

Anti Clock wise formation on right side finger impressions

The Sea waves at Center look like Arch Shape (First stage). The Sea waves at near to the Earth look like Loop Shape (Second stage). The Sea waves touches at Earth look like Whorl Shape (Third stage). The same method follows at Organ construction.

Unnatural:

There is no difference in first stage between natural and unnatural But, natural and unnatural do differ in second stage and third stage. There is difference in second stage and third stage.

Naturally development of organs at womb progresses by cell multiplication and organic molding. This molding/movement of cells takes place in two waves 1.Clockwise and 2.Anti-Clockwise, which is represented by direction of Arch/Loop/Whorl pattern.

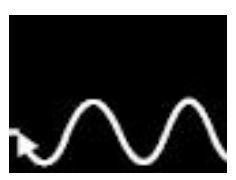

Travelling cells towards left side
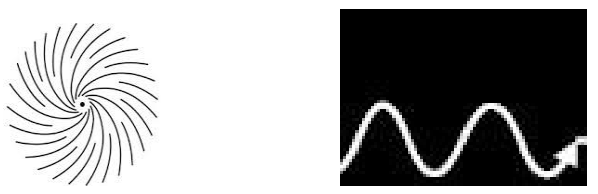

Travelling cells towards right side

After completion of the Organ construction and its linked finger impression are may be one of Arch defense system or Loop defense system or Whorl defense system.

Right Hand Patterns Natural

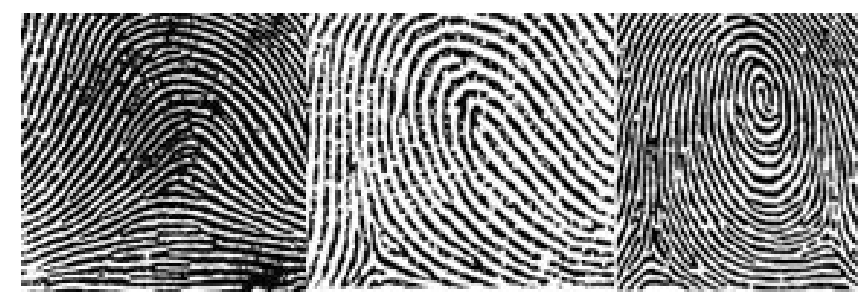


Left Hand Patterns Natural

Right Hand Patterns Unnatural
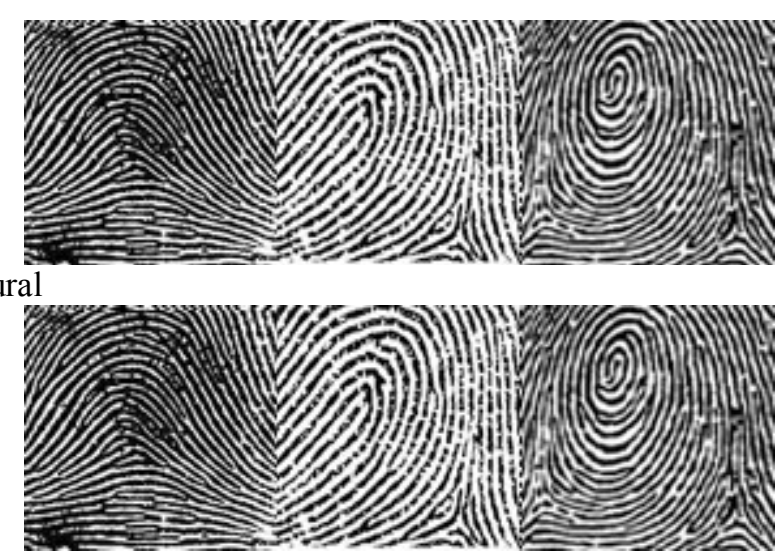

Left Hand Patterns Unnatural

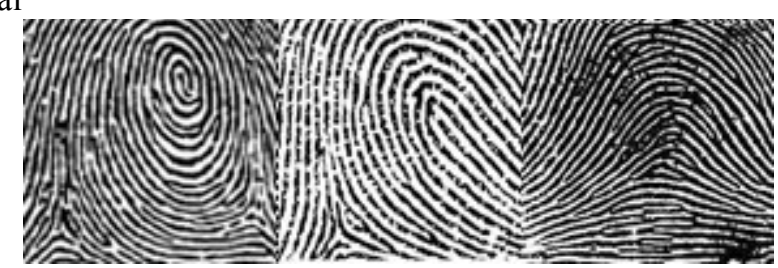

We can't distinguish Arch Patterns where ever they formed. The Ridges run from one side to the other.

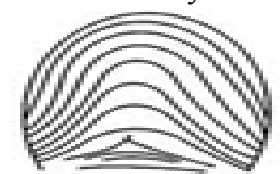

We can't distinguish Whorl Pattern. As they are in clear Circles and Delta to Delta Ridge meets and where we can't distinguish Core formation (formed in the center of the Pattern) whether it is anti clock are clock wise.

Anti Clock Wise

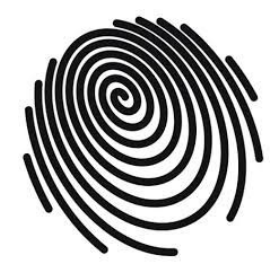

Clock Wise

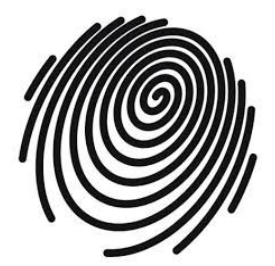

We can distinguish Loop patterns which are available on Right and Left Hands.
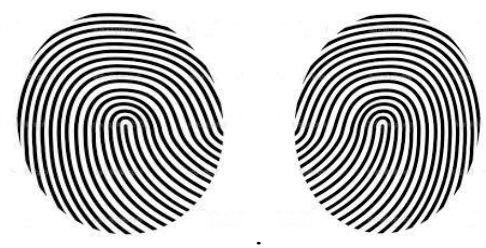

Unnatural Radial (Left) Loop patterns available on Right hand.
Unnatural Radial (Right) Loop patterns Available on left hand 

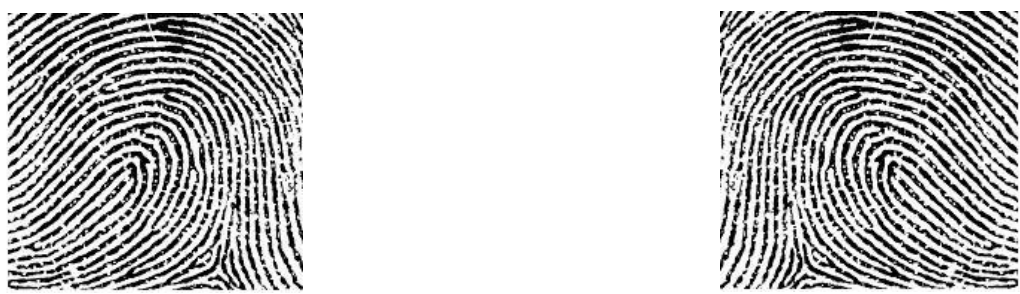

So far we discussed about Natural and Unnatural Pattern formation.

Now we will discuss about Ridge Formation Natural and Unnatural.

Natural Ridge Formation

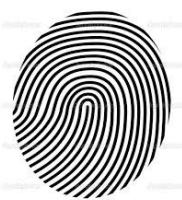

Un Natural Ridge Formation

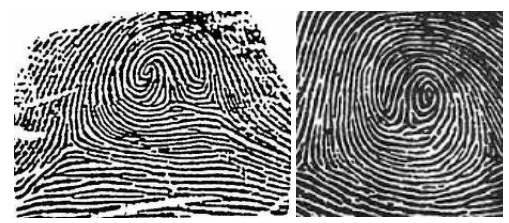

The Organ manufacturing system is depends on the accumulation of Cells, making an Organ either proper way or in proper way. Proper way means the accumulation of Cells construction right side and left side or one and the same.

In proper way means the accumulation of Cells construction right side and left side may be difference. The construction can be understood by verifying the finger impressions.

\section{Hierarchy / Strength}

\section{Finger Impressions}

Arch lowers than the strength of Loop.

Loop lowers than the strength of Whorl.

Whorl has higher strength.

So likewise any patterns found one side of the finger is lower than the other side then the strength of the organ is one side lower than the other side.

Both side same patterns then the strength of the organ equal in both sides.

Both side found Arch patterns the strength of then the organ have minimum strength.

Both side found Loop patterns then the strength of the organ have general strength.

Both side found Whorl patterns then the strength of the organ have maximum strength.

If any different pattern found in one side then the strength of the organ that side strength, can be decided by the pattern.

First Phalange

1. Right and Left thumb related and linked with Brain.

2. Right and Left index related and linked with Bladder/Sex Organs.

3. Right and Left middle related and linked with Kidneys.

4. Right and Left ring related and linked with Pancreas.

5. Right and Left little related and linked with Heart.

6. The down at V Shape between thumb and index related and linked with Stomach.

7. The down at V Shape between index and middle related and linked with Liver.

8. The down at V Shape between middle and ring related and linked with Spleen.

9. The down at V Shape between ring finger and little finger related and linked with Lung. 


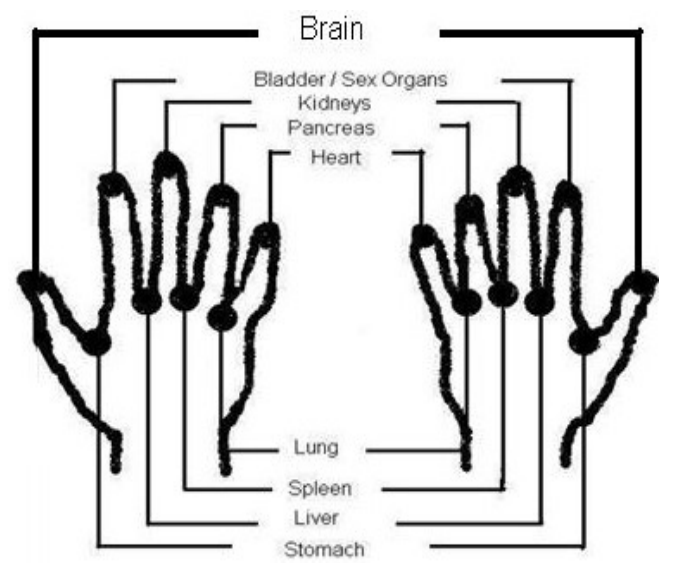

If patterns are same in both sides then the strength of the organ equal in both sides. Else difference in strength.

\section{Arch Family and its Patterns.}

\section{Describing About The Finger Impression Families}

No problem with Plain Arch.

Organ is in weak which Organ is having this type of pattern.

Other Patterns in Arch family is in trouble that we can analyze Organ strength by verifying the finger impressions.

\section{Loop Family and its Patterns.}

Strength can be decided as per the Ridge Count. (Ridge count from delta to core). The strength increases from one Ridge count to another Ridge count.

No problem with Plain Loop.

Other Patterns in Loop family is in trouble that we can analyze Organ strength by verifying the finger impressions.

\section{Whorl Family and its Patterns.}

Difference between plain Whorl and other Whorls.

No problem with Plain Whorl.

There is no problem with Delta to Delta where ridge meets.

There is no problem with Clear Circles.

Other Patterns in Whorl family is in trouble that we can analyze Organ strength by verifying the finger impressions both (Right and Left) fingers.

\section{Research Work}

I have done research in the above subject and got good results.

First of all I have divided Finger Impressions in to Three Families.

Arch Family

Loop Family

Whorl Family

The entire finger Impressions can be classified in to above families only.

Further the strength of the Organs can be decided through finger impressions and the stages of strength are as follows.

\section{Stage-1}

All fingers have Whorl patterns.

Stage-2

Nine Whorls + (One Loop or One Arch)

Stage-3

Eight Whorls + (Two Loops or Two Arches or mixed with Arches and Loops)

Stage-4

Seven Whorls + (Three Loops or Three Arches or mixed with Loops and Arches)

Stage-5

Six Whorls + (Four Loops or Four Arches or mixed with Loops and Arches)

Stage-6 
Five Whorls + (Five Loops or Five Arches or mixed with Loops and Arches)

Stage-7

Four Whorls + (Six Loops or Six Arches or mixed with Loops and Arches)

Stage-8

Three Whorls + (Seven Loops or Seven Arches or mixed with Loops and Arches)

Stage-9

Two Whorls + (Eight Loops or Eight Arches or mixed with Loops and Arches)

Stage-10

One Whorl + (Nine Loops or Nine Arches or mixed with Loops and Arches

Stage-11

All fingers have Loop patterns.

Stage-12

Nine Loops $+($ One Whorl or Arch).

Stage-13

Eight Loops + (Two Whorl or Two Arches or mixed with Whorl and Arches)

Stage-14

Seven Loops + (Three Whorl or Three Arches or mixed with Whorl and Arches)

Stage- 15

Six Loops + (Four Whorl or Four Arches or mixed with Whorl and Arches)

Stage-16

Five Loops + (Five Whorl or Five Arches or mixed with Whorl and Arches)

Stage-17

Four Loops + (Six Whorls or six Arches or mixed with Whorl and Arches)

Stage-18

Three Loops + (Seven Whorl or Seven Arches or mixed with Whorl and Arches) Stage-19

Two Loops + (Eight Whorl or Eight Arches or mixed with Whorls and Arches)

Stage-20

One Loop + (Nine Whorls or Nine Arches or mixed with Whorls and Arches)

Stage-21

All fingers have Arch patterns.

Stage-22

Nine Arches + (One Whorl or One Loop)

Stage-23

Eight Arches + (Two Whorls or Two Loops or mixed with Whorl and Loops)

Stage-24

Seven Arches + (Three Whorl or Three Loops or mixed with Whorl and Loops)

Stage- 25

Six Arches + (Four Whorl or Four Loops or mixed with Whorl and Loops)

Stage-26

Five Arches + (Five Whorl or Five Loops or mixed with Whorl and Loops)

Stage-27

Four Arches + (Six Whorls or Six Loops or mixed with Whorl and Loops)

Stage-28

Three Arches + (Seven Whorl or Seven Loops or mixed with Whorl and Loops)

Stage-29

Two Arches + (Eight Whorl or Eight Loops or mixed with Whorls and Loops)

Stage-30

One Arch + (Nine Whorls or Nine Loops or mixed with Whorls and Loops)

Statistical Data:

1000 Fingerprint Slips Analyzed

Out of 1000

10 have Brain Problem - 1\%

10 have Heart Problem - 1\%

10 have Kidney Problem - 1\%

10 have Pancreas Problem - 1\%

50 have Bladder / Sex Organs Problem - 5\% 
Pancreas

Each and every organ in our body has a function of FILTER.

The friction skin also working as filter to filter discharges.As per the Organs requirement Pancreas releasing Insulin. If consumption of insulin more then we need external insulin to be taken. Called as Diabetic.

\title{
Exceptions
}

Pancreas is clean but not producing insulin as required by the organs why?

Ex: Pancreas is clean but it is failed to produce sufficient insulin to survive Human Organ after extraordinary disease or higher problem faced by the Body.

All Human Organs required Insulin to daily surveillance.

Demerits

1. If Organs weak in condition including Pancreas then we need extra strength from outside. Releasing Insulin is depends on strength of Pancreas. As per the strength Pancreas releases Insulin.

2. If Pancreas weak it will release lower insulin which is lower than the requirement.

What is a Fingerprint?

\section{Questioner}

Fingerprint Expert answer.

Answer-Fingerprint is a pattern formed on the first phalange of finger with ridges and furrows as a design to distinguish design.

Fingerprint Analyzer Answer

The Central Organ covered by the Layers (Ridges) and indexing at first phalange of fingers as just they are reference of the Human Organs.

Core:

Fingerprint Expert Says

Core of the Fingerprint.

Fingerprint Analyzer Says

Central part of the Human Organ.

Ridges:

Fingerprint Expert Says

The skin in the palmer surfaces and soles of the feet is traversed by many elevated and depressed lines. The elevated lines are called ridges.

Fingerprint Analyzer says

The skin in the palmer surfaces and soles of the feet is traversed by many elevated and depressed lines. The elevated lines are indexes of the Layers (covered by the Central Organ).

Pattern: Delta:

Fingerprint Expert says

Whenever an inter space is left between the boundaries of different system of ridges, it is fitted by a small system of its own, which will have some shape called as pattern.

Fingerprint Analyzer says

Whenever an inter space is left between the boundaries of different system of ridges, it is fitted by a small system of its own, which will have some shape, the starting point of the shape is called as Delta.

\author{
With Regards \\ Venkateswara Rao Madduru \\ Fingerprint Analyzer \\ venkatfpb@gmail.com healthbybirth@gmail.com www.healthbybirth.com
}

